10. Isserles, M., et al. Clinical Effectiveness and Tolerability of Electroconvulsive Therapy in Patients with Neuropsychiatric Symptoms of Dementia. J Alzheimers Dis 2017; 57: 45-51

11. Van der Linde, R., et al. Longitudinal course of behavioural and psychological symptoms of dementia: systematic review. B J Psych 2016; 209: 366-377.

\title{
303 - Residential aged care staff's perception of and response to behavioral and psychological symptoms of dementia - a qualitative analysis of clinical care notes
}

Willem S. Eikelboom ${ }^{1,2}$, Jana Koch ${ }^{1,3}$, Elizabeth Beattie ${ }^{4}$, Nicola Lautenschlager ${ }^{5}$, Colleen

Doyle $^{6}$, Kaarin J. Anstey ${ }^{1,3}$, Esther van den Berg ${ }^{2}$, Janne M. Papma ${ }^{2}$, Moyra E. Mortby ${ }^{1,3}$

${ }^{1}$ Neuroscience Research Australia, Sydney, NSW, Australia

${ }^{2}$ Department of Neurology, Erasmus MC University Medical Center, Rotterdam, The Netherlands

${ }^{3}$ School of Psychology, University of New South Wales, Sydney, NSW, Australia

${ }^{4}$ Queensland University of Technology, Brisbane, QLD, Australia

${ }^{5}$ University of Melbourne, Melbourne, VIC, Australia

${ }^{6}$ National Aging Research Institute, Melbourne, VIC, Australia

Keywords: BPSD; dementia; qualitative research; nursing home

Topic: BPSD

Background: Behavioral and psychological symptoms of dementia (BPSD) are very prevalent among individuals with dementia living in residential aged care. The development and implementation of new non-pharmacological interventions to reduce BPSD requires knowledge on the current perception and clinical practice of the care staff. We analyzed clinical care notes to examine the way residential aged care staff reported and managed BPSD in their daily clinical practice.

Methods: We examined semi-structured care notes relating to the presentation and management of behaviors of 76 older residents (67\% female; aged $67-101 ; 75 \%$ with formal dementia diagnosis) prior to participating in the Australian BPSDPLUS Program. As part of standard clinical practice in three residential aged care facilities, staff document the presentation and management of behaviors amongst residents. Using an inductive thematic analytical approach, we analyzed the reported data in the one and a half months prior to commencing participation in the BPSDPLUS Program. Care notes were coded and analyzed by two independent assessors and they discussed themes until consensus was reached.

Results: A total of 465 behavior charts were completed in the one and a half months prior to the commencement of the BPSDPLUS Program. The number of behavioral charts varied widely across residents (Mean=7.3, range 0-93). Behaviors such as refusal of care, repetitive verbal behaviors, and wandering were most often mentioned, while apathy and affective and psychotic symptoms were seldomly reported. When confronted with BPSD, the clinical care notes indicated that care staff tend to respond in a reactive manner by reassuring, redirecting, or distracting the resident. Furthermore, it seems that staff did not routinely investigate potential underlying causes of the BPSD.

Conclusions: These results suggest that the residential care staff primarily detected and responded to externalizing behaviors, while more internalizing behaviors were not reported. Potential underrecognition of internalizing behaviors, as well as the fact that the staff did not routinely examine causes of BPSD are vital observations for the development and implementation of nonpharmacological interventions and care programs targeting BPSD in residential aged care.

304 - Prevalence of and factors related to eating problems in people with dementia Hanxiao WANG, MSN; Siman Chen, MSN; Huangliang WEN, MSN; Jialan Wu, MSN; Xiaoyan Liao, PhD 